\title{
SECAGEM E ARMAZENAMENTO DE SEMENTES DE JUÇARA ${ }^{1}$
}

\author{
Cibele Chalita Martins², Marilene L.A. Bovi³, João Nakagawa² e Carla Gomes Machado ${ }^{4}$ \\ RESUMO - Com o objetivo de verificar o efeito da secagem parcial e do armazenamento sobre a germinação \\ e vigor de sementes de Euterpe edulis obtidas em três épocas de produção, o lote 1 foi colhido em 10 plantas- \\ matriz (PM), em 02/99; o lote 2 em 15 PM, em 04/00; e o lote 3 em 11 PM, em 08/00. Os frutos foram \\ despolpados e as sementes, colocadas para secar por zero, 20 e $40 \mathrm{~h}$ (três sublotes), em câmara seca (temperatura \\ de $27^{\circ} \mathrm{C}$ e umidade relativa de $\left.35 \%\right)$. Os três sublotes foram armazenados a $10^{\circ} \mathrm{C}$, em sacos plásticos $(20$ \\ $\mathrm{mm}$ de espessura) fechados. Durante o armazenamento, a qualidade das sementes foi avaliada a cada seis semanas, \\ por 30 semanas, por meio das seguintes determinações: teor de água $\left(105 \pm 3^{\circ} \mathrm{C} / 24 \mathrm{~h}\right)$, porcentagem de germinação \\ e vigor (primeira contagem e índice de velocidade de germinação). $\mathrm{O}$ aumento do tempo de secagem resultou \\ em sementes com teor de água decrescente, em torno de 14 a $21 \%$ dos valores iniciais, que se mantiveram \\ praticamente inalterados durante o armazenamento. A germinação e vigor das sementes foram prejudicados \\ pela secagem parcial e pelo aumento do tempo de armazenamento, de forma diferenciada entre as épocas \\ de produção, e ambas as características dependem das condições climáticas vigentes durante o desenvolvimento \\ e maturação das sementes.
}

Palavras-chave: Euterpe edulis, conservação e palmito.

\section{DRYING AND STORAGE OF Euterpe edulis SEEDS}

\begin{abstract}
The objective of this research was to verify the effects of partial drying and storage duration on the germination and vigor of three $\boldsymbol{E}$. edulis seed lots developed under different weather conditions. Mature fruits were harvested in the palm collection from the Instituto Agronômico, located in Ubatuba, SP., forming three seed lots. Seeds from ten plants were harvested on 02/99 and constituted the Seed lot 1; seed lot 2 was composed of seeds of fifteen plants collected in 04/00, whereas seeds from eleven plants harvested on 08/00 composed seed lot 3. The fruits were depulped and the seeds were dried for 0,20 and 40 hours, using a drying chamber regulated at $27^{\circ} \mathrm{C}$ of temperature and $35 \%$ of relative humidity. The seeds were stored at $10^{\circ} \mathrm{C}$, in closed plastic bags (20 mm thick). During storage, seed quality was evaluated every six weeks, using the following traits: seed water content $\left(105 \pm 3^{\circ} \mathrm{C} / 24 \mathrm{~h}\right)$, germination (\%) and vigor (first germination counting and index of the velocity of germination (IVG). Seed drying prior to storage decreased the seed water content in $14-21 \%$ of the initial values. Germination and vigor were hampered by partial drying and by the storage duration in a differential mode, and both traits depended on the climatic conditions observed during seed developing and maturation.
\end{abstract}

Keywords: Euterpe edulis, conservation and heart-of-palm.

\section{INTRODUÇÃO}

A espécie Euterpe edulis Mart. vem sendo explorada de forma extrativista e predatória ao longo dos anos, na Floresta Tropical Atlântica do Brasil, para a obtenção do palmito, produto valorizado nos mercados interno e externo. Adicionalmente, a presença de grandes centros urbanos em regiões limítrofes tem favorecido a derrubada da floresta e a ocupação ilegal das áreas para a construção civil, agricultura e pecuária. Desse modo, a maioria das populações de juçara tem sido reduzida devido à exploração indiscriminada e à destruição de seu habitat,

\footnotetext{
${ }^{1}$ Recebido em 05.10.2007 e aceito para publicação em 29.05.2009.

${ }^{2}$ Departamento de Produção Vegetal da Universidade Estadual Paulista Júlio de Mesquita Filho(UNESP). E-mail : <cibele@ fca.unesp.br>.

${ }^{3}$ Pesquisadora Científica, Centro de Horticultura, Seção de Plantas Tropicais IAC/SAA, Campinas-SP “in memorian”.

${ }^{4}$ Programa de Pós-Graduação em Agronomia da UNESP.
} 
onde muitas plantas são derrubadas antes de atingir a maturidade e produzir sementes, único método de propagação da espécie (DRASFIELD et al., 1988).

A procura por sementes de E. edulis para o cultivo comercial, como planta ornamental, e para a implantação de programas de recomposição de matas nativas tem aumentado, gerando demanda crescente por tecnologias favoráveis à produção, qualidade e conservação dessas sementes. No entanto, as sementes de juçara são recalcitrantes, ou seja, sementes de baixa longevidade, sensíveis à desidratação e ao armazenamento nas temperaturas subzero (ANDRADE e PEREIRA, 1997).

Os estudos de armazenamento de sementes de E. edulis indicam que este deve ser realizado em embalagens plásticas para evitar a desidratação e a consequente perda de viabilidade (BOVI et al., 1987; NODARI et al., 1998) e em temperaturas que podem variar de $3{ }^{\circ} \mathrm{C}$ a $20^{\circ} \mathrm{C}$ (BOVI et al., 1987; FIGLIOLIA et al., 1987; ANDRADE e PEREIRA, 1997; NODARI et al., 1998; ANDRADE, 2001).

Dependendo do lote e da temperatura adotada é possível, assim, manter a germinação de sementes de E. edulis em taxas superiores a $50 \%$ por períodos que variam de quatro meses e meio até oito meses (FIGLIOLIAet al., 1987; ANDRADE, 1994; ANDRADE, 2001). O comportamento diferenciado no armazenamento de lotes de uma espécie florestal com sementes recalcitrantes pode estar relacionado ao habitat de origem, à diversidade genética e ao ponto de maturidade fisiológica no momento da colheita (FARRANT et al., 1988; ELLIS etal., 1991; MARTINS et al., 2007). Aqualidade inicial da semente também está entre os fatores que afetam a sua conservação e dependem das condições ambientais vigentes durante a formação, maturação e colheita (CARVALHO e NAKAGAWA, 2000).

As sementes recalcitrantes apresentam teores de água definidos como críticos, abaixo dos quais a viabilidade é reduzida. Apresentam, também, teores letais de água relacionados à perda total da viabilidade (PROBERT e LONGLEY, 1989; PRITCHARD, 1991; HONG e ELLIS, 1992; BILIA et al., 1998; MARTINS et al., 1999abc).

O conhecimento dos teores crítico e letal de água de uma espécie é indispensável para o planejamento e execução da secagem, bem como do armazenamento das sementes. Para sementes de E. edulis a faixa crítica de teor de água está situada entre 39 e $46 \%$ e a letal, entre 16 e $21 \%$, dependendo das características genéticas do lote (MARTINS et al., 1999a). No entanto, a manutenção das sementes com altos teores de água (próximos àqueles do estádio de colheita) durante o armazenamento favorece a germinação dentro da embalagem e o ataque de microrganismos (BOVI et al., 1987; FIGLIOLIA et al., 1987; ANDRADE, 1994; NODARI et al., 1998). Além disso, o armazenamento de sementes recalcitrantes em bandejas na temperatura ambiente e em germinador é inviável (LIMAS et al., 2007). Essas características dificultam a conservação, recomendando-se a semeadura imediata de sementes de E. edulis após a colheita (BOVI e SPIERING, 1989), o que limita a época de semeadura. No Estado de São Paulo, a maturação de frutos dessa espécie é mais frequente entre os meses de abril e agosto (BOVI e SPIERING, 1989), época inadequada para a formação de mudas devido ao déficit hídrico e à baixa temperatura, comuns no período.

Segundo Barbedo e Marcos Filho (1998), a conservação de sementes recalcitrantes poderia ser favorecida por métodos que propiciassem a limitação, ao máximo possível, do crescimento do eixo embrionário, mantendo-se a semente com fornecimento de água suficiente para evitar sua desidratação abaixo do teor de água crítico. Resultados de pesquisas com sementes de Theobroma cacao L., Hevea brasiliensis M. Arg., Inga uruguensis Hook. Et Arn., Myrciaria dubia (H.B.K.) McVaugh e Euterpe oleracea Mart., também recalcitrantes, indicam que a utilização de uma técnica de secagem parcial das sementes pode favorecer a manutenção da qualidade destas durante o armazenamento (HOR et al., 1984; CHIN, 1994; BILIA et al., 1998; GENTIL, 2003; NASCIMENTO, 2006). Diante do exposto, o objetivo do trabalho foi verificar a qualidade das sementes de E. edulis obtidas em três épocas de produção e o efeito da secagem inicial parcial e do tempo de armazenamento sobre a germinação e vigor dessas sementes.

\section{MATERIAL E MÉTODOS}

Frutos maduros de palmito juçara foram coletados manualmente na Estação Experimental do Instituto Agronômico de Campinas (IAC), localizada no Município

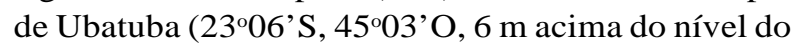
mar), Estado de São Paulo, em épocas distintas de produção, formando três lotes. O primeiro lote (L1) foi composto por sementes colhidas em 10 plantas- 
matriz, em 23/02/99; o segundo (L2), por sementes coletadas em 15 plantas-matriz, em 14/04/00; e o terceiro (L3), por sementes coletadas em 11 plantas-matriz, em 24/08/00. As condições climáticas vigentes no local durante a formação dos frutos estão apresentadas na Figura 2.

Imediatamente após a colheita, cada lote foi transportado em embalagem de polietileno até o Laboratório de Análise de Sementes do Departamento de Produção Vegetal da Faculdade de Ciências Agronômicas da UNESP-Campus de Botucatu, SP, onde os frutos foram despolpados por meio de fricção contra uma peneira de malha de aço, sob água corrente. As sementes malformadas, danificadas ou abaixo do tamanho médio, foram eliminadas. As sementes foram colocadas para secar por 0,20 e 40 h (três sublotes) em câmara seca (temperatura de $27{ }^{\circ} \mathrm{C}$ e umidade relativa de $35 \%$ ), visando à obtenção de três teores distintos de água (valores médios de 48, 43 e 39\%). Após a secagem, os três sublotes foram armazenados no escuro a $10{ }^{\circ} \mathrm{C}$, em sacos plásticos ( $20 \mathrm{~mm}$ de espessura) fechados (MARTINS et al., 2004), dos quais as amostras foram retiradas periodicamente para os testes. Aqualidade das sementes foi avaliada a cada seis semanas até a $30^{\circ}$ semana por meio das seguintes determinações: teor de água - avaliado pelo método da estufa a 105 $\pm 3{ }^{\circ} \mathrm{C} / 24 \mathrm{~h}$ (BRASIL, 1992), utilizando-se quatro subamostras de sete sementes retiradas após o despolpamento, no início da secagem, após cada período de secagem e durante o armazenamento das sementes antes da instalação do teste de germinação; germinação - conduzido em substrato composto por vermiculita esterilizada $\left(120^{\circ} \mathrm{C} / 12 \mathrm{~h}\right)$ na temperatura alternada de $20-30{ }^{\circ} \mathrm{C}$ e luz ( $\left.78 \mathrm{mmol} \mathrm{s}^{-1} \mathrm{~m}^{-2} / 8 \mathrm{~h}\right)$, em caixas plásticas transparentes $(11 \times 11 \times 3,5 \mathrm{~cm})$ do tipo gerbox, utilizando-se quatro subamostras de 20 sementes. O substrato foi umedecido com 1,2 vez o seu peso em água. A contagem das plântulas foi realizada semanalmente do sétimo ao $49^{\circ}$ dia após a semeadura, quando foram calculadas as porcentagens de germinação (plântulas normais); primeira contagem da germinação - realizada aos cinco dias após a semeadura, contabilizando-se o número de botões germinativos emitidos; e índice de velocidade de germinação (IVG) - determinado por meio de adaptação do critério estabelecido por Maguire (1962), contabilizando-se semanalmente as plântulas normais após a instalação do teste.
A análise de variância foi realizada separadamente em cada teste, em delineamento inteiramente casualizado, com quatro repetições, em arranjo fatorial 3 x 6 (tempo de secagem x tempo de armazenamento). A comparação entre as médias dos tratamentos foi efetuada por meio do teste de Tukey a $5 \%$ de probabilidade. Os dados em porcentagem foram transformados em $(x+0,5)^{1 / 2}$ previamente à análise. As médias apresentadas são dos valores originais.

\section{RESULTADOS E DISCUSSÃO}

Em todos os lotes, o aumento do tempo de secagem resultou em sementes com teor de água decrescente (Figura 1) e que permaneceu praticamente inalterado durante o armazenamento: $48,8 \pm 0,7 ; 44,7 \pm 1,1$; e $41,7 \pm 1,1$, respectivamente, no L1 para zero, 20 e $40 \mathrm{~h}$ de secagem; 48,2 $\pm 0,8 ; 42,5 \pm 0,2$; e 37,9 $\pm 0,4$, respectivamente, no L 2 para zero, 20 e $40 \mathrm{~h}$ de secagem; e 46,0 $\pm 0,6 ; 40,7 \pm 0,5$; e 38,2 $\pm 0,6$, respectivamente, no L3 para zero, 20 e $40 \mathrm{~h}$ de secagem, mostrando a adequação da embalagem de polietileno na manutenção dos teores de água relativamente constantes e da sua utilização no armazenamento de sementes recalcitrantes (ANDRADE, 1994; BARBEDOe MARCOS FILHO, 1998; BILIA et al., 1998; ANDRADE, 2001; GENTIL, 2003; MARTINS et al., 2007; NASCIMENTO, 2006).

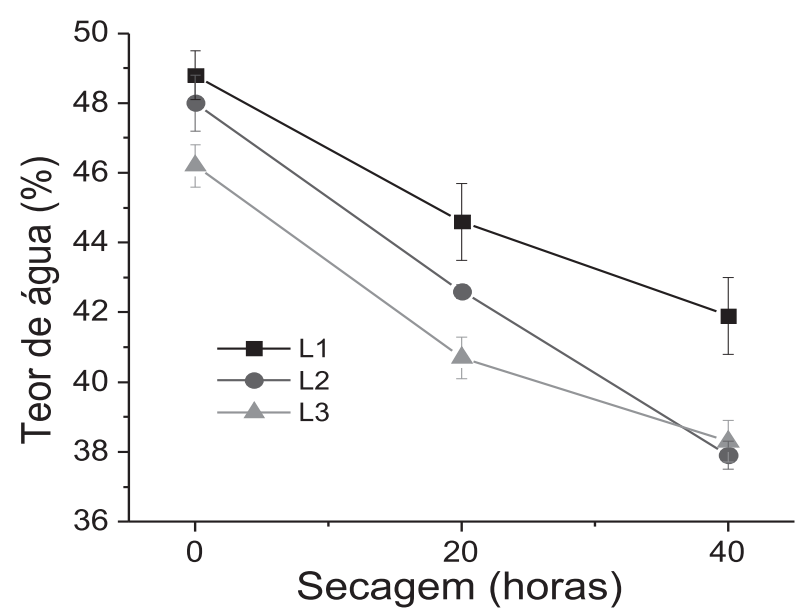

Figura 1 - Teor de água de sementes de três lotes de E. edulis em função do tempo de secagem (barras representam o desvio-padrão da média). Botucatu, UNESP, 1998/ 2000.

Figure 1 - Water content of three seed lots of E. edulis as affected by drying period (bar represents Standard desviation of mean). Botucatu, UNESP, 1998/ 2000 .

R. Árvore, Viçosa-MG, v.33, n.4, p.635-642, 2009 
Os teores de água inicial foram distintos entre as épocas de colheita, com valores de $48,8 \%$ para o L1, colhido em fevereiro; $48 \%$ para o L2, colhido em abril; e 46,2\% para o L3, colhido em agosto. Dados climáticos locais foram obtidos durante o período de formação e maturação dos frutos dos três lotes e mostraram que houve diferenças marcantes entre eles (Figura 2). Dessa forma, observou-se que os teores iniciais de água das sementes mostraram-se dependentes da temperatura média e da evapotranspiração real do mês anterior à colheita (Figuras 1 e 2AE). Observou-se, também, relação positiva entre os dados de precipitação pluviométrica e os teores de água inicial das sementes, especialmente quando comparados os dois últimos meses de maturação dos frutos (Figura 1 e 2B). As perdas no teor de água das sementes devido à secagem (Figura 1) foram menores no L1 (inferiores a 14,2\% após $40 \mathrm{~h}$ de secagem) e mais acentuadas no L2 (superiores a $21 \%$ após o mesmo período). O L3 mostrou valores intermediários, com perda máxima de $17,1 \%$ de água ao final do período de secagem (40 h).

Mesmo após os períodos de secagem, as diferenças no teor de água entre os lotes apresentaram valores considerados elevados. Como E. edulis apresenta semente recalcitrante, muito sensível ao processo de dessecação, a variação no teor de água da semente é também um fator de preocupação que deve influenciar os resultados de germinação.

Os lotes mostraram diferenças também quanto à germinação inicial (Tabela 1). Já os 1 e 3 apresentaram germinação inicial alta, acima de $82 \%$, quando sem secagem e sem armazenamento, enquanto o L2, quando submetido ao mesmo tratamento, a porcentagem de germinação média não passou de $60 \%$.

Tabela 1 - Qualidade das sementes avaliada pelos testes de germinação (G) e de vigor - primeira contagem (PC) e índice de velocidade de germinação (IVG) de três lotes de sementes de E. edulis em função do tempo de secagem e dos períodos de armazenamento (ARM). Botucatu, UNESP, 1998/2000

Table 1 - Seed quality evaluated by tests of germination $(G)$ and vigor - first counting of germination test $(P C)$ and germination rate $(I V G)$ in three seed lots of E. edulis as affected by drying period and storage (ARM). Botucatu, UNESP, 1998/ 2000

\begin{tabular}{|c|c|c|c|c|c|c|c|c|c|c|}
\hline \multirow{2}{*}{$\begin{array}{l}\text { Vigor } \\
\text { (semanas) }\end{array}$} & \multirow[t]{2}{*}{ ARM } & \multicolumn{3}{|c|}{$\begin{array}{c}\text { Lote } 1 \\
\text { Secagem (horas) }\end{array}$} & \multicolumn{3}{|c|}{$\begin{array}{c}\text { Lote } 2 \\
\text { Secagem (horas) }\end{array}$} & \multicolumn{3}{|c|}{$\begin{array}{c}\text { Lote } 3 \\
\text { Secagem (horas) }\end{array}$} \\
\hline & & 0 & 20 & 40 & 0 & 20 & 40 & 0 & 20 & 40 \\
\hline & 0 & $82,5 \mathrm{a} \mathrm{A}$ & $70,0 \mathrm{a} \mathrm{A}$ & $70,0 \mathrm{a} \mathrm{A}$ & $60,0 \mathrm{a} \mathrm{A}$ & $37,5 \mathrm{a} \mathrm{B}$ & $31,3 \mathrm{a} \mathrm{B}$ & $92,5 \mathrm{a} \mathrm{A}$ & $83,8 \mathrm{a} A B$ & $71,3 \mathrm{a} \mathrm{B}$ \\
\hline & 6 & $75,0 \mathrm{a} \mathrm{A}$ & $77,5 \mathrm{a} A$ & $50,0 \mathrm{ab} \mathrm{B}$ & $30,0 \mathrm{~b} \mathrm{~A}$ & $28,8 \mathrm{a} \mathrm{A}$ & $20,0 \mathrm{~b} \mathrm{~A}$ & $61,3 \mathrm{bA}$ & $70,0 \mathrm{a} A$ & $55,0 \mathrm{ab} A$ \\
\hline G & 12 & $77,5 \mathrm{a} \mathrm{A}$ & $67,5 \mathrm{a} A$ & $42,5 \mathrm{ab} \mathrm{B}$ & $15,0 \mathrm{bc} \mathrm{A}$ & $22,5 \mathrm{ab} \mathrm{A}$ & $2,5 \mathrm{c} \mathrm{B}$ & $38,8 \mathrm{~b} \mathrm{~A}$ & $60,0 \mathrm{a} A$ & $47,5 \mathrm{~b} \mathrm{~A}$ \\
\hline \multirow[t]{6}{*}{$(\%)$} & 18 & $70,0 \mathrm{a} \mathrm{A}$ & $70,0 \mathrm{a} \mathrm{A}$ & $25,0 \mathrm{~b} \mathrm{~B}$ & $13,8 \mathrm{bc} \mathrm{AB}$ & $23,8 \mathrm{ab} \mathrm{A}$ & $0,0 \mathrm{c} \mathrm{B}$ & $5,0 \mathrm{c} \mathrm{A}$ & $6,3 \mathrm{~b} \mathrm{~A}$ & $0,0 \mathrm{cA}$ \\
\hline & 24 & $65,0 \mathrm{a} \mathrm{A}$ & $65,0 \mathrm{a} A$ & $27,5 \mathrm{~b} \mathrm{~B}$ & $10,0 \mathrm{c} \mathrm{A}$ & $10,0 \mathrm{~b} \mathrm{~A}$ & $1,3 \mathrm{cA}$ & $0,0 \mathrm{cA}$ & $0,0 \mathrm{c} \mathrm{A}$ & $0,0 \mathrm{cA}$ \\
\hline & 30 & $67,5 \mathrm{a} \mathrm{A}$ & $60,0 \mathrm{a} A B$ & $20,0 \mathrm{~b} \mathrm{~B}$ & $3,8 \mathrm{c} \mathrm{A}$ & $6,3 \mathrm{~b} \mathrm{~A}$ & $0,0 \mathrm{cA}$ & $0,0 \mathrm{c} \mathrm{A}$ & $0,0 \mathrm{c} \mathrm{A}$ & $0,0 \mathrm{c} \mathrm{A}$ \\
\hline & $\mathrm{CV} \%$ & \multicolumn{3}{|c|}{25,7} & \multicolumn{3}{|c|}{60,1} & \multicolumn{3}{|c|}{47,1} \\
\hline & 0 & $40,0 \mathrm{~b} \mathrm{~A}$ & $5,0 \mathrm{a} \mathrm{B}$ & $0,0 \mathrm{a} \mathrm{B}$ & $62,5 \mathrm{a} A$ & $21,3 \mathrm{a} \mathrm{B}$ & $0,0 \mathrm{a} \mathrm{B}$ & $66,3 \mathrm{aA}$ & $15,0 \mathrm{ab} \mathrm{B}$ & $0,0 \mathrm{a} \mathrm{B}$ \\
\hline & 6 & $50,0 \mathrm{ab} \mathrm{A}$ & 7,0a B & $0,0 \mathrm{a} \mathrm{B}$ & $46,5 \mathrm{a} \mathrm{A}$ & $3,8 \mathrm{~b} \mathrm{~B}$ & $0,0 \mathrm{a} \mathrm{B}$ & $55,0 \mathrm{a} \mathrm{A}$ & $20,0 \mathrm{ab} B$ & $0,0 \mathrm{a} \mathrm{C}$ \\
\hline $\mathrm{PC}$ & 12 & $75,0 \mathrm{a} \mathrm{A}$ & $0,0 \mathrm{a} \mathrm{B}$ & $0,0 \mathrm{a} \mathrm{B}$ & $12,5 \mathrm{~b} \mathrm{~A}$ & $1,3 \mathrm{~b} \mathrm{~B}$ & $0,0 \mathrm{a} \mathrm{B}$ & $8,8 \mathrm{~b} \mathrm{~B}$ & $26,5 \mathrm{a} \mathrm{A}$ & $1,3 \mathrm{a} \mathrm{B}$ \\
\hline \multirow[t]{6}{*}{$(\%)$} & 18 & $47,5 \mathrm{ab} \mathrm{A}$ & $0,0 \mathrm{a} \mathrm{B}$ & $0,0 \mathrm{a} \mathrm{B}$ & $16,3 \mathrm{~b} \mathrm{~A}$ & $0,0 \mathrm{~b} \mathrm{~A}$ & $0,0 \mathrm{a} \mathrm{B}$ & $2,5 \mathrm{~b} \mathrm{~A}$ & $6,3 \mathrm{~b} \mathrm{~A}$ & $0,0 \mathrm{a} \mathrm{A}$ \\
\hline & 24 & $65,0 \mathrm{ab} \mathrm{A}$ & $0,0 \mathrm{a} \mathrm{B}$ & $0,0 \mathrm{a} B$ & $6,3 \mathrm{~b} \mathrm{~A}$ & $0,0 \mathrm{~b} \mathrm{~A}$ & $0,0 \mathrm{a} A$ & $0,0 \mathrm{~b} A$ & $0,0 \mathrm{c} A$ & $0,0 \mathrm{a} A$ \\
\hline & 30 & $55,0 \mathrm{ab} \mathrm{A}$ & $0,0 \mathrm{a} \mathrm{B}$ & $0,0 \mathrm{a} \mathrm{B}$ & $3,8 \mathrm{~b} \mathrm{~A}$ & $0,0 \mathrm{~b} A$ & $0,0 \mathrm{a} A$ & $0,0 \mathrm{~b} A$ & $0,0 \mathrm{c} \mathrm{A}$ & $0,0 \mathrm{a} A$ \\
\hline & $\mathrm{CV} \%$ & \multicolumn{3}{|c|}{101,5} & \multicolumn{3}{|c|}{14,0} & \multicolumn{3}{|c|}{82,3} \\
\hline & 0 & $6,8 \mathrm{a} A$ & $5,8 \mathrm{a} A B$ & $3,4 \mathrm{a} B$ & $13,1 \mathrm{a} \mathrm{A}$ & $8,4 \mathrm{a}$ B & $3,6 \mathrm{a} C$ & $16,0 \mathrm{aA}$ & $9,0 \mathrm{a} \mathrm{B}$ & $4,7 \mathrm{a} C$ \\
\hline & 6 & $7,4 \mathrm{aA}$ & $4,8 \mathrm{a} \mathrm{B}$ & $2,0 \mathrm{ab} \mathrm{C}$ & $8,8 \mathrm{~b} \mathrm{~A}$ & $6,3 \mathrm{a} \mathrm{AB}$ & $2,6 \mathrm{~b} \mathrm{~B}$ & $13,5 \mathrm{aA}$ & 7,7a B & $3,5 b \mathrm{C}$ \\
\hline \multirow[t]{5}{*}{ IVG } & 12 & 8,1 a $\mathrm{A}$ & $4,4 \mathrm{a} B$ & $2,0 \mathrm{ab} \mathrm{C}$ & $4,1 \mathrm{c} \mathrm{A}$ & 5,0abc A & $0,3 \mathrm{c} B$ & $4,0 \mathrm{~b} \mathrm{~B}$ & $8,4 \mathrm{a} \mathrm{A}$ & $3,7 \mathrm{ab} \mathrm{B}$ \\
\hline & 18 & $7,1 \mathrm{a} \mathrm{A}$ & $4,3 \mathrm{a} \mathrm{AB}$ & $1,6 a b$ B & $3,5 \mathrm{c} \mathrm{B}$ & $5,8 \mathrm{ab} \mathrm{A}$ & $0,3 \mathrm{c} C$ & $0,9 \mathrm{c} \mathrm{A}$ & $1,6 \mathrm{~b} \mathrm{~A}$ & $0,0 \mathrm{cA}$ \\
\hline & 24 & $7,5 \mathrm{a} \mathrm{A}$ & $4,0 \mathrm{a} \mathrm{B}$ & $0,8 \mathrm{~b} \mathrm{C}$ & $1,9 \mathrm{c} \mathrm{A}$ & $1,8 \mathrm{bc} \mathrm{A}$ & $0,1 \mathrm{c} \mathrm{A}$ & $0,0 \mathrm{cA}$ & $0,0 \mathrm{~b} \mathrm{~A}$ & $0,0 \subset \mathrm{A}$ \\
\hline & 30 & $7,1 \mathrm{a} \mathrm{A}$ & $3,8 \mathrm{a} \mathrm{B}$ & $1,0 \mathrm{~b} \mathrm{~B}$ & $0,8 \mathrm{c} \mathrm{A}$ & $1,0 \mathrm{c} \mathrm{A}$ & $0,0 \mathrm{c} \mathrm{A}$ & $0,0 \subset \mathrm{A}$ & $0,0 \mathrm{~b} \mathrm{~A}$ & $0,0 \subset \mathrm{A}$ \\
\hline & CV\% & \multicolumn{3}{|c|}{28,8} & \multicolumn{3}{|c|}{49,1} & \multicolumn{3}{|c|}{43,3} \\
\hline
\end{tabular}

Para cada característica avaliada, médias seguidas pela mesma letra minúscula na coluna e maiúscula na linha não diferem estatisticamente entre si, pelo teste de Tukey $(\mathrm{P}<0,05)$. As médias apresentadas são dos dados originais. Os dados expressos em porcentagem foram transformados $\mathrm{em}(\mathrm{x}+0,5)^{1 / 2}$.

R. Árvore, Viçosa-MG, v.33, n.4, p.635-642, 2009 
No L1, a secagem por $40 \mathrm{~h}$ reduziu a germinação das sementes armazenadas, principalmente a partir de 18 semanas. Os períodos de secagem de 0 e 20 h não causaram redução significativa da germinação e do vigor avaliado pelo IVG até o final do experimento (30 semanas de armazenamento). No entanto, o IVG teve sensibilidade suficiente para detectar redução gradual do vigor das sementes conforme o período de secagem aumentou. Contrariamente, a primeira contagem de germinação foi mais drasticamente afetada pela secagem, indicando que somente as sementes sem secagem (zero hora) apresentaram vigor significativamente superior às demais, o qual foi mantido, com algumas oscilações, durante todo o período de armazenamento do lote. A redução da germinação e do vigor com a secagem ratifica o comportamento recalcitrante dessa espécie (ANDRADE, 1994; MARTINS et al., 1999a). Em sementes recalcitrantes, a água subcelular está fortemente associada às superfícies macromoleculares, assegurando, em parte, a estabilidade de membranas e macromoléculas. A perda de água estrutural durante o processo de secagem de sementes recalcitrantes causaria alteração de sistemas metabólicos e das membranas celulares, resultando no início do processo de deterioração (FARRANT et al., 1988), que, inicialmente, acarreta redução de vários atributos de performance e vigor da semente e, finalmente, resulta na perda da capacidade germinativa, o que também foi verificado em outros trabalhos envolvendo a secagem de sementes de Euterpe spp (ANDRADE, 1994, MARTINS et al.,1999abc; MARTINS et al., 2004; NASCIMENTO, 2006).

No L2, a germinação e velocidade de germinação (IVG) mostraram que a secagem parcial das sementes por $20 \mathrm{~h}$ possibilitou a manutenção da qualidade inicial da semente até 18 meses, com valores estatisticamente semelhantes ou superiores a 0 ou $40 \mathrm{~h}$ de secagem. Esse desempenho superior no armazenamento das sementes secadas por $20 \mathrm{~h}$ não foi verificado no teste da primeira contagem, que apontou como melhor tratamento as sementes sem secagem, embora isso tenha sido verificado somente até o período de seis semanas de armazenamento.

O efeito favorável da secagem parcial por $20 \mathrm{~h}$ também foi verificado no L3 em todos os testes aplicados. Os resultados da germinação, da primeira contagem e da velocidade de germinação (IVG), mostraram que esse tratamento possibilitou a manutenção da viabilidade das sementes em $60 \%$ por até 12 semanas de armazenamento. Isso parece indicar que esse procedimento favoreceria a conservação da qualidade fisiológica das sementes. Resultados similares foram obtidos por Andrade (1994), também em estudo de armazenamento de sementes de E. edulis, em que a secagem parcial das sementes para teores de água situados entre o da colheita e o crítico favoreceu a manutenção da sua qualidade. Também em sementes de E. oleracea a secagem do lote até $43,4 \%$ do teor de água favoreceu a conservação das sementes, mas somente nas sementes mantidas na temperatura de $20^{\circ} \mathrm{C}$ durante o armazenamento (NASCIMENTO, 2006). No entanto, em sementes de E. espiritosantensis, a secagem parcial desfavoreceu ou não teve efeito sobre a conservação das sementes (MARTINS et al., 2007).

O comportamento diverso no armazenamento entre os lotes de sementes estudados em função da secagem prévia pode ser atribuído a sensibilidades diferenciadas na tolerância à perda de água de acordo com o habitat de origem, a diversidade genética e mesmo à época de colheita, como foi verificado em sementes recalcitrantes de E. espiritosantensis (MARTINS et al., 1999a; MARTINS et al., 2007) e de outras espécies de palmeiras, como Elaeis guineensis (ELLIS et al., 1991), E. edulis e E. oleracea (MARTINS et al.,1999bc) e Bactris gasipaes Kunth (BOVI et al., 2004). Em todos os lotes, com o decorrer do período de armazenamento se constatou um aumento da incidência de fungos nas sementes, dentro das embalagens, e no teste de germinação. Esse fato costuma ocorrer durante o armazenamento de sementes de Euterpe spp devido ao teor de água relativamente alto das sementes e ao processo de deterioração, na ausência de tratamento com fungicidas (BOVI et al., 1987; FIGLIOLIA et al., 1987; ANDRADE, 1994; NASCIMENTO, 2006).

Ao analisar a qualidade inicial dos lotes utilizados no trabalho nos três períodos de secagem, pôde-se verificar que o L3 apresentou sempre a maior porcentagem inicial de germinação (Tabela 1). No entanto, observando o desempenho dos lotes no armazenamento se percebeu que o L1 apresentou maior longevidade, pois mostrou melhor manutenção da sua qualidade fisiológica ao longo do tempo, verificada, principalmente pelo teste de germinação, nos três períodos de secagem.

É interessante observar que o L1 floresceu em época mais amena e completou a maturação em época quente. Nesse período de formação das sementes, a quantidade e intensidade de chuvas foram adequadas, e não houve déficit hídrico no período (Figura $2 \mathrm{abcd}$ ).

R. Árvore, Viçosa-MG, v.33, n.4, p.635-642, 2009 

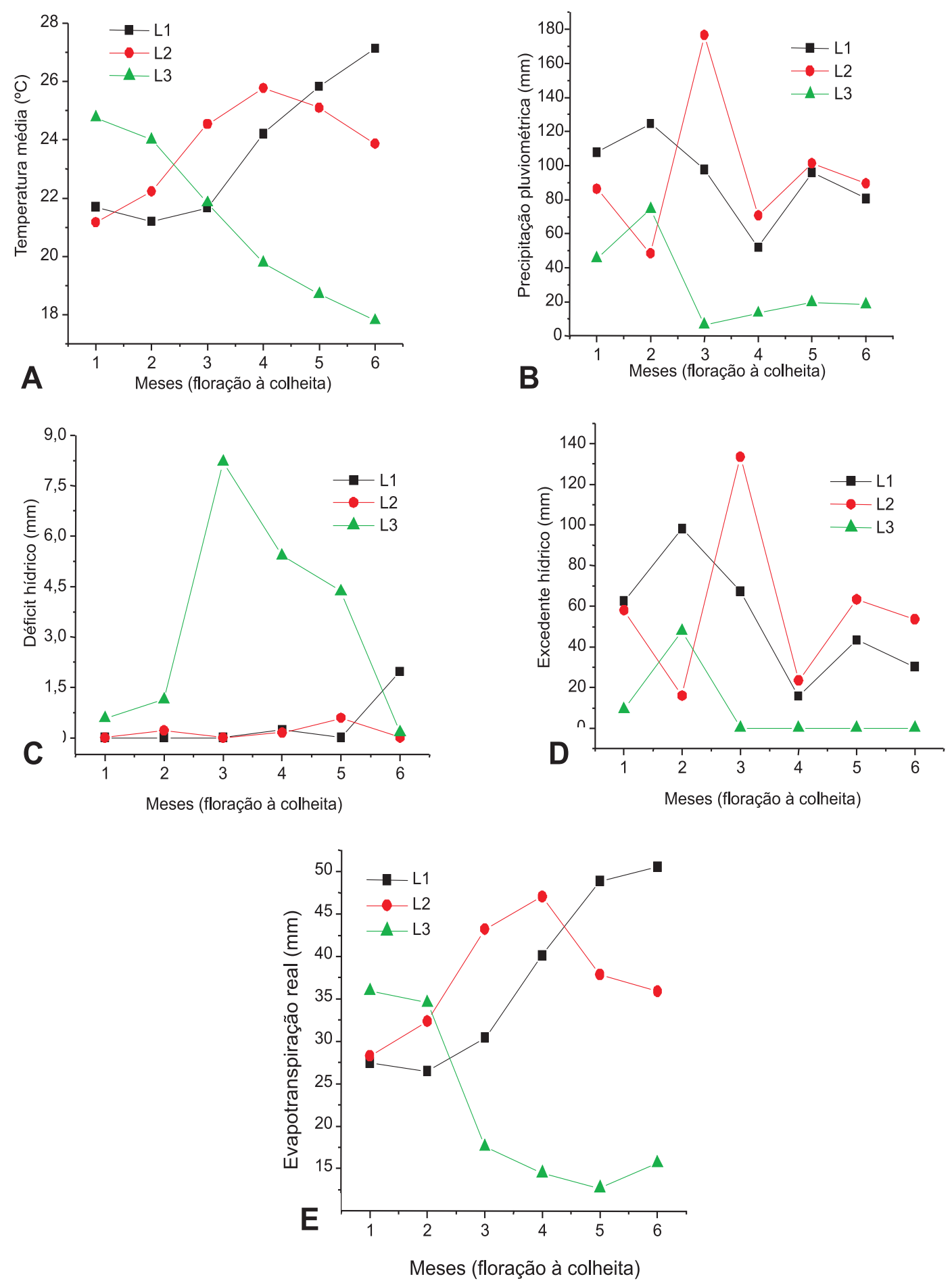

Figura 2 - Temperatura média (A), precipitação (B), déficit (C), excedente hídrico (D) e evapotranspiração real (E) vigente da floração até a colheita dos três lotes de sementes de E. edulis. Ubatuba, IAC 1998/2000.

Figure 2 - Mean temperature, precipitation, water deficit, water excess and actual evapotranspiration occurred from flowering until harvest of the three seed lots of E. edulis. Ubatuba, IAC 1998/2000.

R. Árvore, Viçosa-MG, v.33, n.4, p.635-642, 2009 
O L2 apresentou longevidade intermediária em comparação com os lotes 1 e 3 . Embora esse lote tenha apresentado menor qualidade inicial, mostrou-se mais eficiente que o L3 na manutenção da capacidade de germinação ao longo do tempo. De modo similar ao L1, o lote 2 também floresceu em época de temperaturas amenas e completou a maturação em época quente, embora a temperatura durante a maturação desses tenha sido, em média, um pouco menor que a do L1 (24 e $27^{\circ} \mathrm{C}$, respectivamente). O lote 2 foi formado em uma fase de precipitação intensa no meio da frutificação, cerca de três meses. Assim verificou-se excesso de umidade nesse período (Figura 2abcd).

O L3 com boa performance inicial, especialmente na ausência de secagem, foi o que apresentou mais baixo desempenho no armazenamento, quando perdeu, em maior velocidade, a capacidade de germinação em relação aos demais lotes estudados, e sua longevidade foi de no máximo 18 semanas. A baixa longevidade desse lote parece ter sido causada pelas condições climáticas desfavoráveis vigentes durante a formação e maturação da semente, pois, embora tenha florescido numa época quente e no início da frutificação, também tem ocorrido chuvas em quantidade adequada, e o desenvolvimento dos frutos deu-se em épocas mais amenas e em condições de elevado déficit hídrico. Assim, a formação das sementes do L3 foi mais prejudicada que a dos outros dois lotes, em razão, principalmente, do período de déficit hídrico (Figura 2c), culminando a colheita na época mais fria, condições aparentemente desfavoráveis para uma espécie de Mata Atlântica (Figura 2A).

No Estado de São Paulo, a colheita dos frutos $E$. edulis é mais frequente entre os meses de abril a agosto (BOVI e SPIERING, 1989). Entre os lotes avaliados, o L1 foi o único colhido fora desse período e, também, o único que manteve a germinação inalterada após a secagem por até $40 \mathrm{~h}$, nas sementes sem armazenamento, o que indicaria menor sensibilidade à desidratação que os demais lotes. O L1 foi também o que apresentou maior longevidade. Do ponto de vista ecológico, esse comportamento possibilitaria maior eficiência (longevidade) para superar o período subsequente de falta de água que as sementes produzidas nessa época enfrentariam.

\section{CONCLUSÕES}

Em sementes de Euterpe edulis, o aumento do tempo de secagem resulta em sementes com teores de água decrescentes, mantendo-se praticamente inalterados durante o armazenamento.
A germinação e vigor das sementes são prejudicados pela secagem parcial e pelo aumento do tempo de armazenamento, de forma diferenciada entre as épocas de produção.

\section{AGRADECIMENTO}

À FAPESP pelo financiamento da pesquisa (Programa Jovem Pesquisador).

\section{REFERÊNCIAS}

\section{ANDRADE, A. C. S. Efeito da secagem e do armazenamento sobre a germinação, o vigor de plântulas e a integridade do sistema de membranas em sementes de palmito (Euterpe edulis Mart.). 1994. 87f. Dissertação (Mestrado emFitotecnia) - Universidade Federal do Rio de Janeiro, Rio de Janeiro, 1994.}

ANDRADE, A. C. S. The effect of moisture content and temperature on the longevity of heart of palm seeds (Euterpe edulis M.). Seed Science and Technology, v.29, n.1, p.171-182, 2001.

ANDRADE, A. C. S.; PEREIRA, T. S. Comportamento de armazenamento de sementes de palmiteiro (Euterpe edulis Mart.). Pesquisa Agropecuária Brasileira, v.32, n.10, p.987-991, 1997.

BARBEDO, C. J.; MARCOS FILHO, J. Tolerância à dessecação em sementes. Acta Botânica Brasileira, v.12, n.2, p.145-164, 1998.

BILIA, D. A. C.; MARCOS FILHO, J.; NOVEMBRE, A. D. L. C. Conservação da qualidade fisiológica de sementes de Ingá uruguensis Hook Et Arn. Revista Brasileira de Sementes, v.20, n.1, p.48-54, 1998.

BOVI, M. L. A.; GODOY-JÚNIOR, G.; SAES, L. A. Pesquisas com os gêneros Euterpe e Bactris no Instituto Agronômico de Campinas.

Agronômico, v.39, n.2, p.129-174, 1987.

BOVI, M. L. A.; SPIERING, S. H. Germinação de sementes de palmiteiro. III - Orientação para sua colheita. In: CONGRESSO SOBRE TECNOLOGIA DE SEMENTES FLORESTAIS, 2., 1989, Atibaia. Anais... Atibaia: 1989. p.23-43.

BOVI, M.L.A.; MARTINS, C.C.; SPIERING S.H. Desidratação de sementes de quatro lotes de pupunheira: efeitos sobre a germinação e o vigor. Horticultura Brasileira, v. 22, n. 1, p.109-112, 2004.

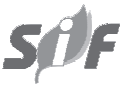

R. Árvore, Viçosa-MG, v.33, n.4, p.635-642, 2009 
BRASIL. Ministério da Agricultura e da Reforma Agrária. Regras para análise de sementes. Brasília: SNDA/DNDV/CLAV, 1992.365p.

CARVAlho, N. M.; NAKAGAWA, J. Sementes: ciência, tecnologia e produção. 4.ed. Jaboticabal: FUNEP, 2000. 588p.

CHIN, H. F. Storage of recalcitrant seeds. In: GOUGH, R.E. Seed quality: basic mechanisms and agricultural implications. New York: Food Products Press, 1994. p.209-222.

DRASFIELD, J.; JOHNSON, D.; SYNGE, H. The palms of the new word: a conservation census. Cambridge: IUCN-WWF Plants Conservation Programme, 1988. 30p.

ELLIS, R. H. et al. Seed storage behaviour in Elaeis guineensis. Seed Science Research, v. 1, n.1, p.9-104, 1991.

FARRANT, J. M.; PAMMENTER, N. W.; BERJAK, P. Recalcitrance - a current assessment. Seed Science and Technology, v.16, n.1, p.155-166, 1988.

FIGLIOLIA, M. B. et al. Conservação de sementes de Euterpe edulis Mart. em diferentes embalagens e ambientes de armazenamento. Boletim

Técnico do Instituto Florestal, v.41, n.1, p.355-368, 1987.

GENTIL, D. F. O. Conservação de sementes de Myrciaria dubia (H.B.K.)

McVaugh. 2003. 41f. Tese (Doutorado

emAgronomia) - Escola Superior de Agricultura Luiz de Queiroz, Piracicaba, 2003.

HONG, T. D.; ELLIS, R. H. Optimum air-dry seed storage environments for arabica coffee. Seed Science and Technology, v.20, n.3, p.547-560, 1992.

HOR, Y. L.; CHIN, H. F.; KARIN, M. Z. The effect of seed moisture and storage temperature on the storability of cocoa (Theoboma cacao) seeds. Seed Science and Technology, v.12, n.2, p.415-420, 1984.

LIMAS, J. D.; SILVA, B. M. S.; MORAES, W. S. Germinação e armazenamento de sementes de Virola surinamensis (Rol.) Warb. (Myristicaceae). Revista Árvore, v.31, n.1, p.37-42, 2007.

R. Árvore, Viçosa-MG, v.33, n.4, p.635-642, 2009
MAGUIRE, J. D. Speed of germination as aid in selection and evaluation for emergence and vigour. Crop Science, v.2, n.2, p.176-177, 1962.

MARTINS, C. C.; NAKAGAWA, J.; BOVI, M. L. A. Dessication tolerance of four seeds lots of Euterpe edulis Mart. Seed Science and Technology, v.28, n.1, p.1-13, 1999a.

MARTINS, C. C. et al. Teores de água crítico e letal para sementes de açaí (Euterpe oleracea Mart. - Palmae). Revista Brasileira de Sementes, v.21, n.1, p.125-132, 1999b.

MARTINS, C. C.; NAKAGAWA, J.; BOVI, M. L. A. Tolerância à dessecação de sementes de palmito-vermelho (Euterpe espiritosantensis Fernandes). Revista Brasileira de Botânica v.22, n.3, p.391-396, 1999c.

MARTINS, C. C. et al. Temporary storage of jussara palm seeds: effects of time, temperature and pulp on germination and vigor. Horticultura Brasileira, v.22, n.2, p.271-276, 2004.

MARTINS, C. C.; NAKAGAWA, J.; BOVI, M. L. A. Qualidade fisiológica de sementes de palmitovermelho em função da desidratação e do armazenamento. Horticultura Brasileira, v.25, n.1, p.188-192, 2007.

NASCIMENTO, W. M. O. Conservação de sementes de açaí (Euterpe oleracea Mart.). 2006. 60f. Tese (Doutorado emAgronomia) - Escola Superior de Agricultura Luiz de Queiroz, Piracicaba, 2006.

NODARI, R. O. et al. Conservação de frutos e sementes de palmiteiro (Euterpe edulis Mart.) sob diferentes condições de armazenamento. Revista Árvore, v.22, n.1, p.1-10, 1998.

PRITCHARD, H. W. Water potential and embryonic axis viability in recalcitrant seeds of Quercus rubra. Annals of Botany, v.67, n.1, p.43-49, 1991.

PROBERT, R. J.; LONGLEY, P. L. Recalcitrant seed storage physiology in three aquatic grasses (Zizania palustris, Spartina anglica and Portesia coarctata). Annals of Botany, v.63, n.1, p.53-63, 1989. 